\title{
Hand Biometrics in Mobile Devices
}

\author{
Alberto de Santos-Sierra ${ }^{1}$, Carmen Sanchez-Avila ${ }^{1}$, Javier Guerra-Casanova ${ }^{1}$ \\ and Aitor Mendaza-Ormaza ${ }^{2}$ \\ ${ }^{1}$ Group of Biometrics, Biosignals and Security \\ Centro de Domótica Integral \\ Universidad Politécnica de Madrid \\ ${ }^{2}$ Universidad Carlos III de Madrid, Leganés \\ Spain
}

\section{Introduction}

New trends in biometrics are inclined to adapt both identification and verification process to mobile devices in order to provide real scenarios and applications with a more secure frame. In fact, upcoming applications related to electronic commerce are demanding more trustworthy and reliable techniques to ensure their operations and transactions Van Thanh (2000), for instance. In other words, biometrics are requested to provide an appropriate alternative to current pin codes and passwords.

Furthermore, commercial biometric systems normally have no constraints in terms of computational cost or involved hardware but they do aim the highest accuracy in personal identification. In contrast, applying biometrics to mobile devices requires a reconsideration of previous lack of constraints since a mobile device is at present far from being comparable to current biometric systems in terms of hardware.

Based on these concerns, this document presents a biometric system based on hand geometry oriented to mobile devices, since hand images were acquired with mobile devices. This approach offers the possibility of identifying individuals easily with a non-intrusive acquisition procedure, using a picture taken with the mobile phone and avoiding the use of a flat surface to place the hand, providing this system with a non-contact characteristic. Moreover, the hand can be acquired without constraints in orientation, distance to camera or illumination, since the proposed technique within this paper is invariant to previous changes. This property provides an increase in the acceptance of the biometric technique by the final user, together with the fact that no removal of rings, watches and the like is required for image acquisition.

In contrast, such lack of constraints in acquisition demands a more challenging solution in relation to segmentation and feature extraction. The former operation must be able to isolate completely hand from background, regardless what is behind the hand. In case of feature extraction, the template must be independent from which hand is considered for identification (left or right hand) and invariant to changes in orientation, position, distance to camera and the like. In addition, the proposed template considers finger widths and lengths and, besides, information from four fingers (index, middle, ring and little/pinky) is considered, instead of global features from the whole hand. 
The proposed system has been tested with three databases collected in different environments, with two mobile phones and therefore different cameras.

First database was created to evaluate the proposed algorithm in terms of detection accuracy, containing samples of 120 individuals from a population with different ages, gender and races, taken with an HTC, throughout a period of four months. Segmentation algorithm is exclusively tested by images in second database, a collection of synthetic hand images, based on first database, but with different environments (soil, grass, tiles, walls and the like), so that real scenarios can be simulated. Finally, third database was collected to evaluate to what extent segmentation and feature extraction algorithm were invariant to different degrees of hand opening, distance to camera and rotation. This latter database was completed using a Sony Ericsson w380i mobile.

The achieved results provide an Equal Error Rate of $4.1 \pm 0.2 \%$ by using 60 features (15 features in each finger) and seven training samples for template extraction, being able to obtain an EER of $3.8 \pm 0.1 \%$ when increasing the number of training samples to ten, by using a Support Vector Machine linear classifier.

The layout of the paper is arranged as follows: Section 2 provides a literature review in hand biometrics, drawing attention to hand geometry approaches. Sections 3 and 4 describe both the segmentation procedure and how features are extracted. Before presenting the results in Section 6, a description of the databases involved to evaluate the biometric system is provided in Section 5. Finally, this document ends with conclusions and future work in Section 7.

\section{Related work}

The distinctive characteristics within the human hand have inspired different identification techniques based mainly on geometric aspects Sanchez-Reillo et al. (2000); Zheng et al. (2007), texture patterns Kong et al. (2009) and hand vein templates Shahin et al. (2008). Considering geometric aspects, there exist several previous works based on a wide variety of topics Singh et al. (2009); Zheng et al. (2007): fingers and hand measurements Sanchez-Reillo et al. (2000); Singh et al. (2009), hand contour de Santos Sierra et al. (2009); Yoruk et al. (2006), 3D geometric representation Kanhangad et al. (2009), graph description Gross et al. (2007); Rahman et al. (2008) and so forth. Furthermore, research lines in hand biometrics based on geometric aspects consider a fusion among different characteristics leading to an enhancement in verification and identification Varchol et al. (2008); Wang et al. (2009); Yang \& Ma (2007).

An aspect of relevance regards how the hand is acquired concerning not only the acquisition devices but also to what extent hand background is under control. Generally, CCD Cameras are the most common device to acquire hand images Covavisaruch \& Prateepamornkul (2006); Sanchez-Reillo et al. (2000); Yu et al. (2009) providing with a wide variety of images resolutions depending on the camera. In addition, scanners are also considered as an adequate alternative to CCD devices Hashemi \& Fatemizadeh (2005); Varchol et al. (2008). For the sake of a precise acquisition, hand is usually located on a flat surface provided with guiding pegs ensuring that hand is exactly placed on the same position. However, some problems arise from this approach which concern shape deformation, an increase in the device acquisition complexity and, more recently, contact-based acquisition devices can be considered controversial regarding hygiene and public-health issues Zheng et al. (2007).

On the basis of this fact, peg-free biometric systems tackles with this problem although many approaches still preserve the flat surface to locate the hand. Some works propose an acquisition procedure avoiding completely any contact with surfaces de Santos Sierra et al. (2009); Zheng et al. (2007). However, these contact-free approaches cope with the problem 
of isolating the hand from a more complicated background, since previous works based on contact or semi-contact devices had a controlled background. In other words, contact-free biometric systems provide less invasiveness in acquisition at expense of an increase in the computational cost of the feature extraction and segmentation algorithm.

Regarding invasiveness, most of previous works require a removal of rings, bracelets and similar complements Kukula et al. (2007), although many trends tend to extract hand features and descriptors without requiring any removal de Santos Sierra et al. (2009); Yoruk et al. (2006).

Different illumination scheme have been proposed. Normally, a gray-scale image provides enough information concerning not only geometric aspects but also palmprint or fingerprint texture information Arif et al. (2006); Yang \& Ma (2007). In contrast, color images provide more information on skin color and therefore more useful information for contact-less approaches. In addition, several color spaces have been also proposed to facilitate the procedure of segmentation, although most common used space is RGB Tan et al. (2009).

Several authors have proposed an infra-red illumination environment Ferrer et al. (2009); Shahin et al. (2008) based on the fact that infra-red illumination allows to extract hand contour easily since infra-red light highlights that region closer to the focus, and therefore, background is rarely illuminated. However, these acquisition systems require both a special illumination and an infra-red camera, difficult to be embedded on daily devices like mobiles and smartphones, for instance.

Hand biometric acceptation was assessed in Kukula \& Elliott (2005; 2006); Kukula et al. (2007) evaluating the performance of the biometric system in relation to the number of attempts in accessing the system. In fact, the repeated used of the device provides an increase in the identification accuracy of participants. Therefore, the individuals get easily habituated to hand biometric devices, although many users required more restricted instructions when facing the system. Similar conclusions were obtained in de Santos Sierra et al. (2009) where hand images were acquired in a free space.

New trends in biometrics tend to adapt current systems to mobile devices. However, not every biometric technique is suitable for this adaptation. Furthermore, mobile devices imply certain limitations in relation to computational cost and performance efficiency and accuracy. Obviously, mobile security is not so demanding as, for instance, an application in an international airport. In the literature, there exist previous approaches concerning biometrics and mobiles involving different biometric characteristics: Face Recognition on Symbian OS Abeni et al. (2006); Ijiri et al. (2006), Voice Recognition Shabeer \& Suganthi (2007), Keystroke Dynamics Saevanee \& Bhatarakosol (2008), Hand de Santos Sierra et al. (2009), Palmprint Han et al. (2007) or Finger Pressure McLoughlin \& Naidu (2009); Saevanee \& Bhatarakosol (2008); Shabeer \& Suganthi (2007). All previous work coincide on the same conclusions: mobile devices imply limitations for biometric accuracy and efficiency, but provide a high degree of security in daily applications.

\section{Segmentation}

As presented in the literature review (Section 2), segmentation in hand biometrics was almost a trivial operation, since the background is completely uniform and different in color and intensity to hand texture Boreki \& Zimmer (2005); Sanchez-Reillo et al. (2000); Yu et al. (2009). However, the acquisition procedure proposed within this document requires a more demanding segmentation procedure able to isolate entirely and precisely hand from 
background. Notice, that this background is unknown and there is no prior information about it, since images could be acquired at any place regardless the environment.

Images were acquired in a RGB color space, which is a standard format for comercial mobiles. However, the proposed segmentation is carried out in a different color space, since RGB provides not enough information to distinguish properly hand from background. In order to obtain an adequate accuracy in segmentation, CIELAB (CIE $1976 \mathrm{~L}^{*}, \mathrm{a}^{*}, \mathrm{~b}^{*}$ ) was selected due to its ability to describe all visible colors by the human eye Gonzalez \& Woods (1992); Mojsilovic et al. (2002); Tan et al. (2009).

This color space transformation facilitates enormly the segmentation operation by offering a representation in which pixels corresponding to skin texture are separated in terms of $\mathrm{L}^{*} \mathrm{a}^{*} \mathrm{~b}^{*}$ intensities from rest pixels. Selecting which layer contains more distinctive information for segmentation is in fact a crucial matter Albin et al. (2002); Gonzalez \& Woods (1992); Recky \& Leberl (2010); Wang et al. (2010). The proposed method makes use of the Entropy of an image, $H$, to select which layer contains more unique or distinguishing information Luengo-Oroz et al. (2010).

Normally, experiments show that layer $a$ provides more distinctive information.

After selecting the proper layer, pixels must be divided into two groups: a group containing pixels corresponding to hand, and a second group gathering those pixels describing background. This classification is carried out by a $k$-means algorithm, which provides a suitable clustering solution for segmentation problem Recky \& Leberl (2010), gathering in a unique cluster those pixels corresponding to hand texture.

Although a deep explanation of $k$-means procedure is far beyond the scope of this article Gonzalez \& Woods (1992), the segmentation problem can be stated as follows: given an image $I$, the aim of this $k$-means algorithm is to divide the image $I$ into $k$ clusters, minimizing the intracluster square sum (Eq. 1):

$$
\underset{\mathbf{S}}{\arg \min } \sum_{i=1}^{k} \sum_{\mathbf{x}_{j} \in S_{i}}\left\|\mathbf{x}_{j}-\boldsymbol{\mu}_{i}\right\|^{2}
$$

where $\mathbf{S}$ corresponds to the segments in which the image $I$ is divided, and $\mu_{i}$ represents the $i^{\text {th }}$ clustering distribution mean.

Classification is based not only in colour information, but also in the position within image. This is essential for avoiding the effects of rings and small ornaments on hands, since they are considered as part of the hand, despite of slightly deforming the hand. However, the aim of this procedure is twofold: to ensure fingers not to be splitted from hand, remaining the hand as a unique solid blob, and to keep simple the segmentation algorithm (the most time consuming step in hand recognition, Section 6), considering both the fact that the procedure could be implemented in mobiles and that ignoring measures extracted from regions associated to rings is easier than correcting the error provided by the ring.

A deeper understanding of the effects produced on the template and system accuracy remains as future work, together with an adequate processing to avoid this effect.

Obviously, this fact affects posterior measures, and therefore, the effects of rings in feature extraction will be explained under Section 4 .

In order to obtain a binary image (those pixels belonging to hand represented by a high value, and thus background represented by zero), $k$ is set to $k=2$. In addition, ensuring which group corresponds to hand (' 1 ' values) or background ('0' values) is easily carried out by analyzing which group is more isolated from the outside boundary (image border). Reader may notice that this assumption implies that individuals have colaborated with the system in locating 
the hand within the camera visual field, and therefore within the boundaries of the image. Otherwise, in case of hand being too close to camera (and therefore not being confined within image boundaries) or hand not appearing completely in the image, a correct segmentation will not be carried out (hand does not appear completely within image), implying that image must be rejected and requiring a new acquisition.

Due to illumination and background, the result provided by the $k$-means algorithm could be slightly inaccurate in the boundary, and therefore a morphological operation must be performed to soften that contour. The selected operation is a morphological opening Gonzalez \& Woods (1992); Luengo-Oroz et al. (2010), with a disk structural element of small dimension (5 pixels of radius), since such a structural element suites adequately hand geometry, based on the rounded shape of a hand, without any sharp contour.

\section{Template extraction}

This section defines the features to be extracted from hand in order to reduce the biometric information contained within the hand to more comparable and measurable parameters. These features must describe and define the hand uniquely and univocally, and must remain invariant to changes of size, distance to camera, rotation and similar variations in acquisition. Some previous works provide similar templates based on width fingers and distances extracted from hand Boreki \& Zimmer (2005); Sanchez-Reillo et al. (2000), and others consider free-space acquisition Ferrer et al. (2009); Zheng et al. (2007), but without considering a high degree of freedom in hand changes and mobile devices acquisition.

Before extracting features, tips and valleys are detected according to previous work de Santos Sierra et al. (2009); Munoz et al. (2010), based on the difference of pixels in the hand contour and hand centroid.

The proposed method extracts features by dividing the finger from the basis to the tip in $m$ parts. Each of these former parts measures the width of fingers, based on the euclidean distance between two pixels. Afterwards, for each finger, the $m$ components are reduced to $n$ elements, with $n<m$, so that each $n$ component contains the average of $\left\lfloor\frac{m}{n}\right\rfloor$ values, gathering mean value, $\mu$ and standard deviation $\sigma$. In other words, template is extracted based on an average of a finger measures set, being more reliable and precise than one single measure (Section 6). This approach provides a novelty if compared to previous works in literature (Section 2), where single measures were considered.

Furthermore, each $n$ component is normalized by the corresponding finger length, in an attempt to provide independence on distance to camera.

Therefore, the template can be mathematically described as follows. Let $F=\left\{f_{i}, f_{m}, f_{r}, f_{l}\right\}$ be the set of possible fingers, namely index, middle, ring and little, respectively. Let $\Lambda=$ $\left\{\lambda_{i}, \lambda_{m}, \lambda_{r}, \lambda_{l}\right\}$ be the set of distances for the corresponding finger.

Each finger $f_{k}$ is divided into $m$ parts from basis to top, resulting in the set of widths $\Omega_{f_{k}}=$ $\left\{\omega_{1}, \ldots, \omega_{m}\right\}$. From set $\Omega$, the template is represented by $\Delta_{f_{k}}=\left\{\frac{\delta_{1}^{f_{k}}}{\lambda_{f_{k}}}, \ldots, \frac{\delta_{k}^{f_{k}}}{\lambda_{f_{k}}}\right\}$, where each $\delta_{t}^{f_{k}}$ is defined as the average value of at least $\left\lfloor\frac{m}{n}\right\rfloor$ components in $\Omega_{f_{k}}$. Notice that this division could imply that last element $\delta_{n}$ could be the average of more than $\left\lfloor\frac{m}{n}\right\rfloor$ components in order to ensure that every element in $\Omega_{f_{k}}$ is considered to create $\Delta_{f_{k}}$.

Features are not extracted in thumb finger due to its variability in terms of movement, position and direction, and thus, none sufficient distinctive information can be extracted, despite of 
normalization. Therefore, the biometric template representing a hand is composed of a total of $4 \times n$ elements. This relation will be studied in detailed within results section (Section 6).

In order to compare templates among individuals, this paper proposes (Support Vector Machines, SVM Kumar \& Zhang (2006; 2007)) with linear kernel functions as an adequate and accurate classifier, which has provided the best results when compared to other classifiers and kernel functions.

The number of samples to create the template in order to train the SVM properly is studied in Section 6.3.

\section{Database acquisition}

This biometric method is oriented to mobile applications and therefore, the algorithm must be tested with images acquired from mobile devices. The databases differ in the mobile device involved to acquire images, number of individuals, images sizes and the like. First database is used to evaluate (train, validate and test) the whole system considering identification efficiency. Second was created synthetically based on first database to evaluate only the performance of segmentation, with the aim of assessing the implemented algorithms in different environments and scenarios. Finally, third database was collected to evaluate feature changes in rotation, hand opening and distance to camera. These databases are available at http://www.gb2s.es.

\subsection{First database}

This database is the most complete containing hand captures of 120 different individuals of an age range from 16 to 60 years old, gathering males and females in similar propotion.

Furthermore, considering a contact-less approach for biometric hand recognition, every hand image was acquired without placing the hand in any flat surface neither requiring any removal of rings, bracelets or watches. Instead, the individual was required to open his/her hand naturally, so the mobile device (an HTC) could take a photo of the hand at 10-15 cm of distance with the palm facing the camera.

This acquisition implies no severe constraints on neither illumination nor distance to mobile camera and every acquisition was carried out under natural light. These approach combines several current challenges in hand biometric recognition with the limitation of mobile devices. Therefore, it is a database with a huge variability in terms of size, skin color, orientation, hand openness and illumination conditions.

In order to ensure a proper feature extraction, independently on segmentation, acquisitions were taken on a defined blue-coloured background, so that segmentation can be easily performed, focusing on hands. This background can be easily replaced by another texture like soil, tiles and the like, as it will be seen in Sections 5.2 and 6.1.

Some samples of this first database are provided in Figure 1.

Both hands were taken, in a total of two sessions: During the first session, 10 acquisitions from both hands are collected; second session is carried out after 10-15 minutes, collecting again 10 images per hand.

The image size provided by the device is $648 \times 338$ pixels.

\subsection{Second database}

Second database is entirely aimed to evaluate segmentation, assessing to what extent the segmentation algorithm can satisfactory perform a hand isolation from background on real scenarios. 

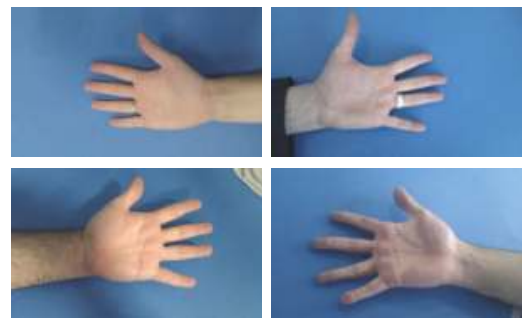

Fig. 1. Samples of first database, with blue-coloured background.

In order to simulate that hand is located over different backgrounds, that region considered as background in the segmentation procedure carried out for images in first database is replaced by different textures. Afterwards, an opening morphological operator (with a disk structural element of radius 5) for colour images Gonzalez \& Woods (1992) is considered to avoid possible edges separating hand and the latter texture, providing a more realistic environment. Different backgrounds are considered in an attempt to cover all possible real scenarios, containing textures from carpets, fabric, glass, grass, mud, different objects, paper, parquet, pavement, plastic, skin and fur, sky, soil, stones, tiles, tree, walls and wood. Five different images from every texture were considered to ensure more realistic environments. All previous texture backgrounds were taken from $h t t p: / / m a y a n g . c o m / t e x t u r e s /$.

Some examples of second database can be seen in Figure 2.
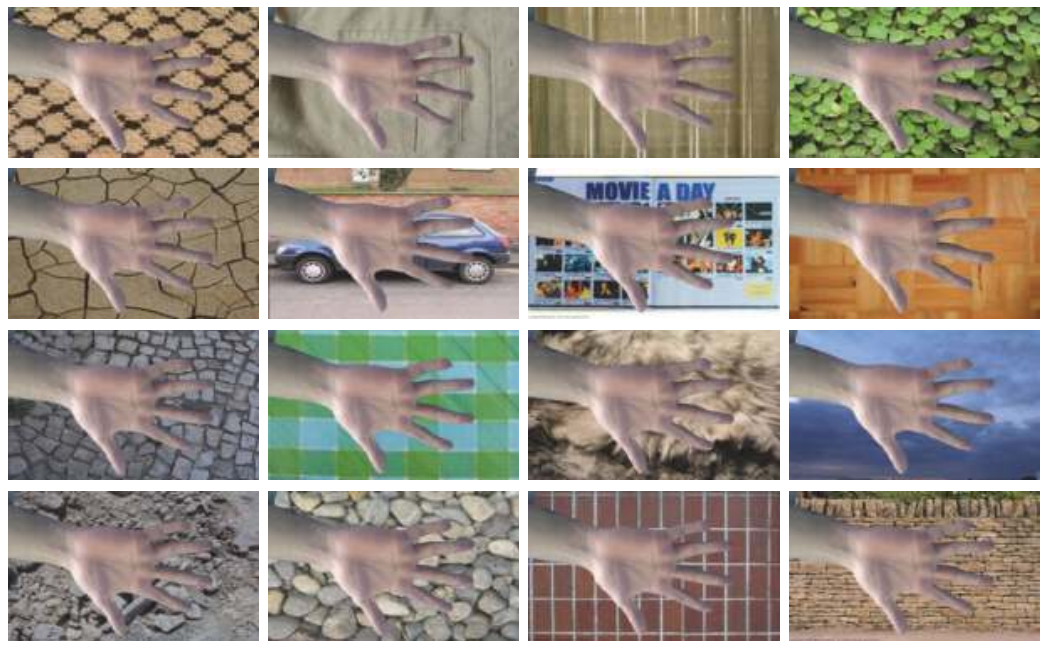

Fig. 2. Samples of second database in different backgrounds for a given acquisition taken from first database.

For each image on first database, a total of $5 \times 17$ (five images and 17 textures) images are created. Therefore, second database collects a total of $120 \times 2 \times 20 \times 5 \times 17=408000$ images (120 individuals, 2 hands, 20 acquisitions per hand, five images and 17 textures) to properly evaluate segmentation on real scenarios. 


\subsection{Third database}

Finally, third database is collected by a Sony Ericcson w380i mobile devices, with a camera of 1.3 Megapixels and image dimensions of $1280 \times 1024$. This database contains images from 10 individuals with the aim of measuring three different aspects: rotation, 50 images per angle were taken $\left(5\right.$ angles: $0^{\circ}, 45^{\circ}, 90^{\circ}, 135^{\circ}$ and $180^{\circ}$. All these angles are referred to the vertical line formed by individual's head and feet, placing the hand with the palm facing the individual with the fingers pointing to the top), for each individual (a total of 2500 images); hand openness, 50 images per position (three possibilities: normal, not very open and very open) and individual which makes a total of 1500 images; and distance to camera, 50 images per distance (two distances: $15 \mathrm{~cm}$ and $30 \mathrm{~cm}$ ) and individual (10 users) making a total of 1000 images. Regarding openness degree, normal degree is considered when the surface of the palm is totally flat, with a openness radius of infinity. Not very open means to have a slightly concave curvature of the palm (a big positive radius of curvature), and very open means to have a slightly convex curvature of the palm (a big radius of curvature, but opposite to the previous one).

Therefore, the database contains 5000 images of 10 individuals. It must be pointed out that angles in this database are not precisely measured but approximated, similarly to the distance to the camera and the hand openness degree. Several samples of this database are provided in Figure 3.
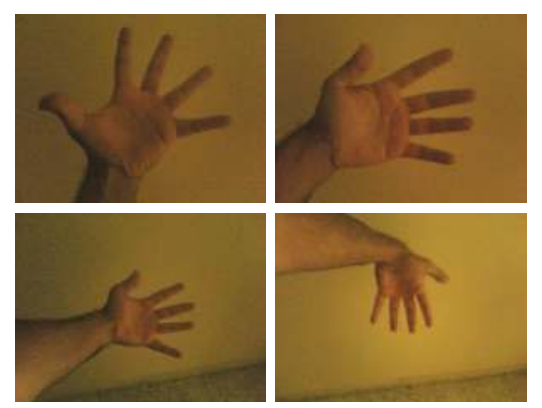

Fig. 3. Samples of third database, with different rotation angles, distance to camera and openness degree.

\section{Results}

Evaluation in hand biometrics involves assessing how the segmentation procedure isolates hand from background, to what extent features are invariant to changes (position, scale or orientation) and the accuracy in identifying and verifying individuals given a database. Therefore, this section will be divided into three different parts corresponding to each aspect to be assessed.

\subsection{Segmentation evaluation}

Concerning segmentation evaluation, a supervised evaluation method Munoz et al. (2010); Zhang et al. (2008) was considered, comparing the segmentation result to a ground-truth solution obtained based on the segmentation carried out for first database. This first database contains hand acquisitions with a known background, becoming relatively easy to extract 


\begin{tabular}{cc|cc|cc}
\hline \hline Texture & $F(\%)$ & Texture & $F(\%)$ & Texture & $F(\%)$ \\
\hline Carpets & $80.7 \pm 0.3$ & Paper & $86.2 \pm 0.1$ & Stones & $82.4 \pm 0.1$ \\
Fabric & $83.2 \pm 0.1$ & Parquet & $76.1 \pm 0.2$ & Tiles & $89.2 \pm 0.2$ \\
Glass & $92.5 \pm 0.2$ & Pavement & $88.3 \pm 0.3$ & Tree & $98.7 \pm 0.1$ \\
Grass & $91.6 \pm 0.1$ & Skin and Fur & $86.7 \pm 0.1$ & Wall & $87.3 \pm 0.3$ \\
Mud & $82.6 \pm 0.2$ & Sky & $89.3 \pm 0.1$ & Wood & $83.8 \pm 0.2$ \\
Objects & $88.2 \pm 0.3$ & Soil & $71.1 \pm 0.2$ & & \\
\hline
\end{tabular}

Table 1. Segmentation evaluation by means of factor $\mathrm{F}$ in a synthetic database with 17 different background textures.

precisely hand from background. This segmentation will be considered as ground-truth for this evaluation.

In order to measure to what extent the result is similar to ground-truth, factor $F$ Alpert et al. (2007) provides a suitable manner to assess segmentation. Factor $F$ is defined by Eq. 2:

$$
F=\frac{2 R P}{R+P}
$$

where $R$ (Recall) represents the number of true positives (true segmentation, i.e. classify a pixel corresponding to hand as hand) in relation to the number of true positives and false positives (false background segmentation, i.e. consider a pixel corresponding to background as hand) and $P$ (Precision) represents the number of true positives in relation to the number of true positives and false negatives (false hand segmentation, i.e. consider a pixel corresponding to hand as background).

The results of factor $F$ obtained for second database are presented in Table 1 . Notice that those textures similar in color and textures to hand (like mud, wood, skin and parquet) decrease the performance of the segmentation algorithm.

In addition, we present the segmentation result within Figure 4, where first row provides some examples of both left and right hands from first database, together with their segmentation results in second row, representing the ground-truth segmentation. Besides, some examples from the synthetic database were taken to compare segmentation results between an under-control background and their corresponding synthetic images, with a random background (third and four row). A complete understanding of the effects of these backgrounds on identification rates will be a future work aim.

\subsection{Feature invariance evaluation}

Providing information on the evaluation regarding feature extraction represents a difficult task, since the assessment would consist of comparing each feature in different situations for each database. However, a small sample of features (representative of all possible features) is considered and are compared using the fifth database. These features correspond to those more close to tip, i.e. $\frac{\delta_{n}^{f_{k}}}{\lambda_{f_{k}}}$, assuming that the variation of these features is similar to the rest of features along fingers.

Therefore, the evaluation will consider only three aspects: different degrees of hand opening, distance to camera and rotation.

Firstly, this study only considers three degrees of opening: Original position (standard position), a not very open hand where the individual is indicated to close slightly the hand 

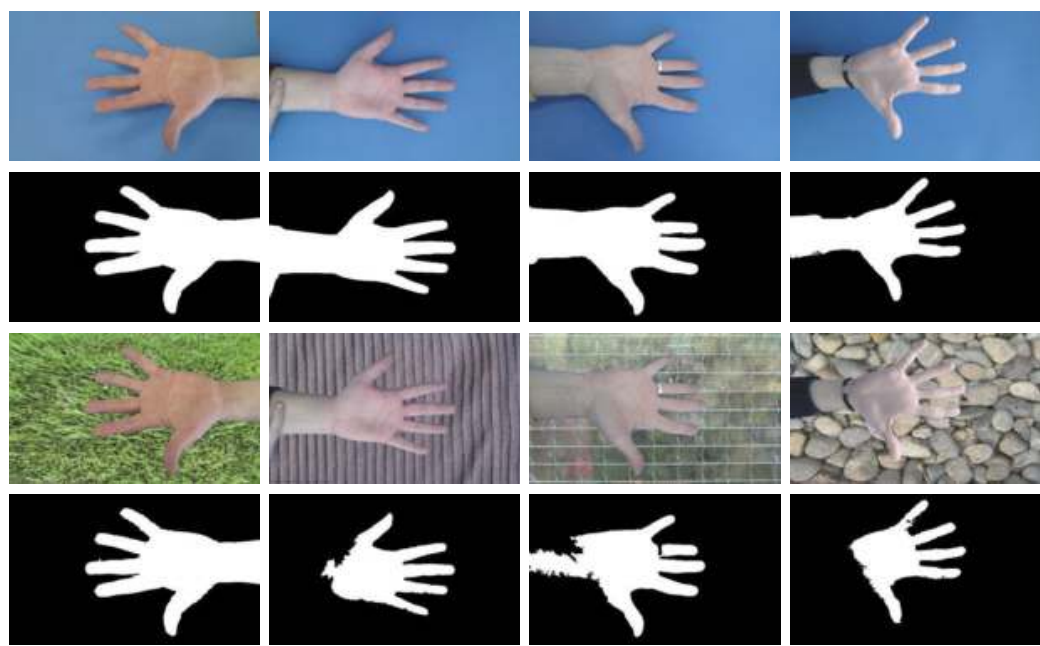

Fig. 4. Segmentation Evaluation: First row, original first database; Second row, the corresponding segmentation result (ground-truth); Third row, the associated synthetic images with different backgrounds; Fourth row, the segmentation result for synthetic images.

\begin{tabular}{cccc}
\hline \hline \multicolumn{3}{c}{ Feature Original } & Not very open Very open \\
\hline Index & $1.2 \pm 0.1$ & $1.1 \pm 0.2$ & $1.5 \pm 0.1$ \\
Middle & $1.4 \pm 0.2$ & $1.3 \pm 0.1$ & $1.7 \pm 0.2$ \\
Ring & $1.3 \pm 0.1$ & $1.4 \pm 0.2$ & $1.6 \pm 0.1$ \\
Little & $1.7 \pm 0.1$ & $1.7 \pm 0.1$ & $1.9 \pm 0.1$ \\
\hline
\end{tabular}

Table 2. Variation of a certain feature in each finger for different openness degrees. The results correspond to the mean difference value and their dispersion.

and a very open hand degree where the subject is supposed to open entirely the hand, Figure 3.

Table 2 contains the difference in terms of pixels between extracted features to a feature reference set, containing on the first column the differences among hand acquisitions with no changes in hand openness. This experiment is repeated 1000 times selecting randomly the feature reference set, presenting thus the main statistics (difference mean and deviation) in Table 2. This procedure is the same in posterior Tables 3 and 4 .

These results highlight that although there is no significative variation in terms of difference average and deviation, there is a slight variation when the hand is entirely open. This is due to the fact that opening the hand extensively can deform to some extent the geometry of the fingers, but this variation is not significative if compared to original values (first column).

Secondly, the variation of the features is studied in relation to the distance between hand and mobile. According to fifth database, only two distances were considered: standard distance (15 cm approx.) and far distance (30 cm approx.). Notice that a very short distance to the mobile camera makes the hand not fit the mobile screen. Table 3 shows how the distance affects moderately the error between features, although the variation is comparable to original deviation, and it is possible to affirm that extracted features are invariant to distance to camera. 


\begin{tabular}{ccc}
\hline \hline Feature Original (15 cm approx.) & $30 \mathrm{~cm}$ approx. \\
\hline Index & $1.2 \pm 0.1$ & $1.5 \pm 0.3$ \\
Middle & $1.4 \pm 0.2$ & $1.8 \pm 0.2$ \\
Ring & $1.3 \pm 0.1$ & $1.9 \pm 0.3$ \\
Little & $1.7 \pm 0.2$ & $2.1 \pm 0.2$ \\
\hline \hline
\end{tabular}

Table 3. Variation of a certain feature in each finger for two distances to camera. The results correspond to the mean difference value and their dispersion.

\begin{tabular}{cccccc}
\hline \hline Feature Original & $45^{\circ}$ & $90^{\circ}$ & $135^{\circ}$ & $180^{\circ}$ \\
\hline Index & $1.2 \pm 0.1$ & $1.4 \pm 0.2$ & $1.3 \pm 0.1$ & $1.1 \pm 0.1$ & $1.4 \pm 0.1$ \\
Middle & $1.4 \pm 0.2$ & $1.5 \pm 0.1$ & $1.2 \pm 0.2$ & $1.3 \pm 0.2$ & $1.3 \pm 0.2$ \\
Ring & $1.3 \pm 0.1$ & $1.5 \pm 0.2$ & $1.6 \pm 0.1$ & $1.4 \pm 0.1$ & $1.5 \pm 0.2$ \\
Little & $1.7 \pm 0.2$ & $1.8 \pm 0.1$ & $1.5 \pm 0.2$ & $1.8 \pm 0.1$ & $1.7 \pm 0.1$ \\
\hline \hline
\end{tabular}

Table 4. Variation of a certain feature in each finger for different rotation angles. The results correspond to the mean difference value and their dispersion.

Finally, Table 4 provides information on the property of invariance for extracted features. There is no significative change in feature difference when compared to original, which means that features are invariant to rotation.

Moreover, a practical manner of assessing whether features are invariant to changes is indicated by the global Equal Error Rate (EER) provided in next subsection (Section 6.3). Notice that first database contains a wide range of cases with different values in position, orientation and distance to camera.

Finally, there exist other factors worthy of study, and which remain as future work, like blur effects in image, since it is very common that images acquired by a mobile phone are blurred due to small movements of the camera when obtaining the picture.

\subsection{System accuracy, EER}

Previous sections have provided an evaluation in terms of segmentation and feature extraction. However, the most important aspect regards the capability of the biometric system to identify or verify individuals. The evaluation of the biometric accuracy involves again a wide number of elements such as the database, the number of samples and features used to train the system. Thereby, a deep understanding of these former factors is required to obtain the best results in identification/verification.

In contrast, this section will only consider two aspects covering those main problems that general biometric systems cope with: 1) The relation between accuracy and number of features; 2) The dependency of the whole biometric system (in terms of Equal Error Rate, EER Sanchez-Reillo et al. (2000)) in relation to the number of samples required to train the system.

The first study is carried out by using the first database (Section 5), fixing the number of training samples $(T=7)$ and testing samples $(U=13)$, being assessed by a $K$-fold cross validation approach. Samples from first session in the database were used as training samples, using acquisitions from second session as testing samples. For simplicity sake, five values for $n$ were considered: $\{5,7,10,12,15\}$. Changes due to smaller variations in $n$ are negligible. Furthermore, only one hand is considered (left or right) in identification, selected by the individual from which hand was taken. A fusion of both hands recognition could improve 


\begin{tabular}{cccccc}
\hline \hline & $n=5$ & $n=7$ & $n=10$ & $n=12$ & $n=15$ \\
\hline EER (\%) with $\Delta_{n}$ & $16.4 \pm 0.1$ & $14.1 \pm 0.2$ & $8.3 \pm 0.3$ & $5.7 \pm 0.3$ & $4.1 \pm 0.1$ \\
EER (\%) with $\Omega_{m=n}$ & $18.3 \pm 0.2$ & $15.2 \pm 0.1$ & $9.1 \pm 0.2$ & $7.2 \pm 0.2$ & $6.8 \pm 0.1$ \\
\hline \hline
\end{tabular}

Table 5. Variation of Equal Error Rate (EER, \%) in relation to the number of features, $n$.

\begin{tabular}{ccccc}
\hline \hline Training Samples & $T=3$ & $T=5$ & $T=7$ & $T=10$ \\
\hline EER (\%) with $\Delta_{n}$ & $14.2 \pm 0.4$ & $8.4 \pm 0.2$ & $4.1 \pm 0.2$ & $3.8 \pm 0.1$ \\
EER (\%) with $\Omega_{m=n}$ & $15.4 \pm 0.2$ & $10.1 \pm 0.3$ & $6.8 \pm 0.1$ & $6.1 \pm 0.2$ \\
\hline \hline
\end{tabular}

Table 6. Variation of Equal Error Rate (EER, \%) in relation to the number of training samples, $T$.

the overall accuracy, but lacks of interest for a final application in mobiles. Nonetheless, this system allows users to provide any of both hand for identification, so that individuals should not remember with which hand were enrolled.

The results obtained under this experimental layout are presented in Table 5, where EER (\%) is provided in relation to $n$ for both the proposed approach based on average values $\left(\Delta_{n}\right)$ and the traditional approach, based on single fingers width $\left(\Omega_{m=n}\right)$.

Besides, reader may notice that the same number of samples are extracted from each finger, although it could be possible that some fingers contribute differently to the final pattern. A deeper understanding of this idea remains as future work, (Section 7).

Similarly, the relation between EER and the number of training samples is of interest, since a compromise must be achieved between this two previous parameters. Notice that an application based on a high number of training samples will cause a rejection from final users due to its obvious inconvenience. To this end, Table 6 is provided, employing samples from first session in the database (ten samples $T=10$ ) to train and samples from second session to test $(U=10$ samples, concretely). The experimental result is obtained setting the feature extraction parameters to $n=15$, employing a $K$-fold cross validation approach. Obviously, the higher the number of elements in training, the higher the system accuracy. However, a modest variation in terms of EER is observed with $T \geq 7$, being $T=7$ the selected value which gathers a compromise between accuracy and comfortability (number of training samples). In addition, a comparison to a traditional approach $\left(\Omega_{m=n}\right)$ is also provided in Table 6 .

\subsection{Mobile implementation}

The presented system has been implemented on two different arquitectures: a MATLAB implementation to be run in a PC computer @2.4 GHz Intel Core 2 Duo with 4GB 1067 $\mathrm{MHz}$ DDR3 of memory and a JAVA-Android implementation oriented to an HTC Desire with @1GHz and 576 MB RAM. Reader can notice obvious differences in hardware, and therefore the implementation of this approach must be tackled with different perspectives in each situation.

Table 7 provides a comparative study of the speed performance of each implementation. Although HTC implementation is more time-consuming, it takes less than 3 seconds to identify individuals, which is very suitable for daily applications. Temporal values in former Table 7 were obtained by measuring both implementations average performance by using first database. 


\begin{tabular}{ccc}
\hline \hline Process & PC @2.4GHz (seconds) HTC @1GHz (seconds) \\
\hline Acquisition & $<0.1$ & $<0.1$ \\
Segmentation & $0.5 \pm 0.07$ & $1.3 \pm 0.1$ \\
Feature Extraction & $0.3 \pm 0.1$ & $0.7 \pm 0.1$ \\
Matching & $0.1 \pm 0.02$ & $0.4 \pm 0.06$ \\
\hline
\end{tabular}

Table 7. Comparative temporal study of implementations in PC (second column) and HTC mobile (third column) measured in seconds

\section{Conclusions and future work}

This document presents a biometric system based on hand geometry oriented to mobile devices. This system incorporates some novel and challenging aspects since images are acquired without no severe constraints in terms of illumination, position, distance to camera and orientation; acquisitions were taken with cameras embedded on commercial mobile devices, providing thereby low resolution images lacking in details and precision; and no flat surface is required to locate the hand or pegs to force a certain position to the hand.

Due to all these previous characteristics, a non-invasive biometric system comes up gathering not only comfortability to the final user (take a hand picture with the mobile) but also reliance on the performance of the biometric system, being able to identify individuals with an EER of $4.1 \pm 0.2 \%$ with seven training samples and a total of 60 features $(4 \times n$ with $n=15)$ and seven training samples. Moreover, an EER of $3.8 \pm 0.1 \%$ can be obtained by increasing the number of training samples to ten images.

In addition, this biometric system has been seriously evaluated covering every main aspect in a biometric system: segmentation, feature extraction and identification rate. The evaluation relies on three databases, which are publicly available on $h t t p: / / w w w . g b 2 s . e s$ collecting a wide range of samples with the purpose of assessing previous aspects, considering different devices, environment conditions, situations, backgrounds, population and the like.

The obtained results come up with an important conclusion: the proposed extracted features yield to an independence to changes in image acquisitions.

Furthermore, a study concerning invariance to blur operations will be contemplated. Blur and fuzzy effects deserve special attention since they simulate the behavior of a moved acquisition, something very common in mobile acquisitions due to the low quality acquisition system. Despite of building a new database, this effects will be reproduced with different image processing algorithms. In addition, a deeper understanding on the contribution of each individual feature in relation to final accuracy will be also considered, together with a fusion scheme with palmprint.

With the aim of a mobile device application, several details must be improved. First of all, it is desirable to reduce the number of training samples, preserving the accuracy. Secondly, an adaptive SVM is supposed to decreased EER throughout time, decreasing the number of false rejections (situations that exasperate the final user). Thirdly, a PCA algorithm could obtain the principal components in the extracted templates, reducing the number of features within the pattern. Furthermore, a study on the device independence of the biometric system will lead to make possible the fact of enrolling an individual with one device and accessing with other, yielding to multiple applications.

In addition, reader may notice that this system entails, at least, more than one individual in order to carry out a comparison. This situation barely happens on a mobile device, since they are not shared by more than one individual. Therefore, how would it be possible to keep 
rates without knowing others bimetric data? This biometric system provides a solution for a centralized access system, where the accesing devices are mobile apparatus. Individuals can have access to their corresponding mobiles, by being verified using this biometric system. However, if this biometric system is used offline, i.e. without accessing previous centralized system, the biometric algorithm must be able to identify the individual without being compared to others. This can be achieved by storing fake templates in mobile, or providing a one-class SVM. In any case, this situation involves a final scenario and a final implementation and, therefore, it has little relation to biometric topics, despite of being a challenging problem regular to all biometric systems applied to daily applications.

Finally, an adaptation to current biometric standards ISO/IEC JTC1/SC37 will be also considered.

\section{References}

Abeni, P., Baltatu, M. \& D'Alessandro, R. (2006). Nis03-4: Implementing biometrics-based authentication for mobile devices, Global Telecommunications Conference, 2006. GLOBECOM '06. IEEE, pp. 1-5.

Albin, S., Rougeron, G., Peroche, B. \& Tremeau, A. (2002). Quality image metrics for synthetic images based on perceptual color differences, Image Processing, IEEE Transactions on 11(9): $961-971$.

Alpert, S., Galun, M., Basri, R. \& Brandt, A. (2007). Image segmentation by probabilistic bottom-up aggregation and cue integration, IEEE Conference on Computer Vision and Pattern Recognition, 2007. CVPR '07., pp. 1-8.

Arif, M., Brouard, T. \& Vincent, N. (2006). Personal identification and verification by hand recognition, Engineering of Intelligent Systems, 2006 IEEE International Conference on, pp. 1-6.

Boreki, G. \& Zimmer, A. (2005). Hand geometry: a new approach for feature extraction, Automatic Identification Advanced Technologies, 2005. Fourth IEEE Workshop on, pp. 149-154.

Covavisaruch, N. \& Prateepamornkul, P. (2006). Personal identification system using hand geometry and iris pattern fusion, Electro/information Technology, 2006 IEEE International Conference on, pp. 597-602.

de Santos Sierra, A., Guerra Casanova, J., Sánchez Ávila, C. \& Jara Vera, V. (2009). Silhouette-based hand recognition on mobile devices, 43rd Annual 2009 International Carnahan Conference on Security Technology, 2009., pp. 160-166.

Ferrer, M., Fabregas, J., Faundez, M., Alonso, J. \& Travieso, C. (2009). Hand geometry identification system performance, Security Technology, 2009. 43rd Annual 2009 International Carnahan Conference on, pp. 167-171.

Gonzalez, R. C. \& Woods, R. E. (1992). Digital Image Processing, Addison-Wesley Longman Publishing Co., Inc., Boston, MA, USA.

Gross, R., Li, Y., Sweeney, L., Jiang, X., Xu, W. \& Yurovsky, D. (2007). Robust hand geometry measurements for person identification using active appearance models, Biometrics: Theory, Applications, and Systems, 2007. BTAS 2007. First IEEE International Conference on, pp. 1-6.

Han, Y., Tan, T., Sun, Z. \& Hao, Y. (2007). Embedded palmprint recognition system on mobile devices, ICB, pp. 1184-1193. 
Hashemi, J. \& Fatemizadeh, E. (2005). Biometric identification through hand geometry, Computer as a Tool, 2005. EUROCON 2005.The International Conference on, Vol. 2, pp. 1011-1014.

Ijiri, Y., Sakuragi, M. \& Lao, S. (2006). Security management for mobile devices by face recognition, Mobile Data Management, 2006. MDM 2006. 7th International Conference on, pp. $49-49$.

Kanhangad, V., Kumar, A. \& Zhang, D. (2009). Combining 2d and 3d hand geometry features for biometric verification, Computer Vision and Pattern Recognition Workshops, 2009. CVPR Workshops 2009. IEEE Computer Society Conference on, pp. 39-44.

Kong, A., Zhang, D. \& Kamel, M. (2009). A survey of palmprint recognition, Pattern Recogn. 42(7): 1408-1418.

Kukula, E. \& Elliott, S. (2005). Implementation of hand geometry at purdue university's recreational center: an analysis of user perspectives and system performance, Security Technology, 2005. CCST '05. 39th Annual 2005 International Carnahan Conference on, pp. 83-88.

Kukula, E. \& Elliott, S. (2006). Implementation of hand geometry: an analysis of user perspectives and system performance, Aerospace and Electronic Systems Magazine, IEEE 21(3): 3-9.

Kukula, E., Elliott, S., Gresock, B. \& Dunning, N. (2007). Defining habituation using hand geometry, Automatic Identification Advanced Technologies, 2007 IEEE Workshop on, pp. 242-246.

Kumar, A. \& Zhang, D. (2006). Personal recognition using hand shape and texture, Image Processing, IEEE Transactions on 15(8): 2454-2461.

Kumar, A. \& Zhang, D. (2007). Hand-geometry recognition using entropy-based discretization, Information Forensics and Security, IEEE Transactions on 2(2): 181-187.

Luengo-Oroz, M. A., Faure, E. \& Angulo, J. (2010). Robust iris segmentation on uncalibrated noisy images using mathematical morphology, Image Vision Comput. 28: 278-284. URL: http://portal.acm.org/citation.cfm?id=1663651.1663753

McLoughlin, I. \& Naidu, N. (2009). Keypress biometrics for user validation in mobile consumer devices, Consumer Electronics, 2009. ISCE '09. IEEE 13th International Symposium on, pp. $280-284$.

Mojsilovic, A., Hu, H. \& Soljanin, E. (2002). Extraction of perceptually important colors and similarity measurement for image matching, retrieval and analysis, Image Processing, IEEE Transactions on 11(11): 1238 - 1248.

Munoz, A.-C., de Santos Sierra, A., Ávila, C., Casanova, J., del Pozo, G. \& Vera, V. (2010). Hand biometric segmentation by means of fuzzy multiscale aggregation for mobile devices, Emerging Techniques and Challenges for Hand-Based Biometrics (ETCHB), 2010 International Workshop on, pp. 1-6.

Rahman, A., Anwar, F. \& Azad, S. (2008). A simple and effective technique for human verification with hand geometry, Computer and Communication Engineering, 2008. ICCCE 2008. International Conference on, pp. 1177-1180.

Recky, M. \& Leberl, F. (2010). Windows detection using k-means in cie-lab color space, Pattern Recognition (ICPR), 2010 20th International Conference on, pp. 356 -359.

Saevanee, H. \& Bhatarakosol, P. (2008). User authentication using combination of behavioral biometrics over the touchpad acting like touch screen of mobile device, Computer and Electrical Engineering, 2008. ICCEE 2008. International Conference on, pp. 82 -86. 
Sanchez-Reillo, R., Sanchez-Avila, C. \& Gonzalez-Marcos, A. (2000). Biometric identification through hand geometry measurements, Pattern Analysis and Machine Intelligence, IEEE Transactions on 22(10): 1168-1171.

Shabeer, H. \& Suganthi, P. (2007). Mobile phones security using biometrics, Conference on Computational Intelligence and Multimedia Applications, 2007. International Conference on, Vol. 4, pp. $270-274$.

Shahin, M., Badawi, A. \& Rasmy, M. (2008). A multimodal hand vein, hand geometry, and fingerprint prototype design for high security biometrics, Biomedical Engineering Conference, 2008. CIBEC 2008. Cairo International, pp. 1-6.

Singh, A., Agrawal, A. \& Pal, C. (2009). Hand geometry verification system: A review, Ultra Modern Telecommunications Workshops, 2009. ICUMT '09. International Conference on, pp. 1-7.

Tan, W., Wu, C., Zhao, S. \& Chen, S. (2009). Hand extraction using geometric moments based on active skin color model, Intelligent Computing and Intelligent Systems, 2009. ICIS 2009. IEEE International Conference on, Vol. 4, pp. 468-471.

Van Thanh, D. (2000). Security issues in mobile ecommerce, Database and Expert Systems Applications, 2000. Proceedings. 11th International Workshop on, pp. $412-425$.

Varchol, P., Levicky, D. \& Juhar, J. (2008). Multimodal biometric authentication using speech and hand geometry fusion, Systems, Signals and Image Processing, 2008. IWSSIP 2008. 15th International Conference on, pp. 57-60.

Wang, W.-C., Chen, W.-S. \& Shih, S.-W. (2009). Biometric recognition by fusing palmprint and hand-geometry based on morphology, Acoustics, Speech and Signal Processing, 2009. ICASSP 2009. IEEE International Conference on, pp. 893-896.

Wang, X., Huang, X. \& Fu, H. (2010). A color-texture segmentation method to extract tree image in complex scene, Machine Vision and Human-Machine Interface (MVHI), 2010 International Conference on, pp. $621-625$.

Yang, F. \& Ma, B. (2007). Two models multimodal biometric fusion based on fingerprint, palm-print and hand-geometry, Bioinformatics and Biomedical Engineering, 2007. ICBBE 2007. The 1st International Conference on, pp. 498-501.

Yoruk, E., Konukoglu, E., Sankur, B. \& Darbon, J. (2006). Shape-based hand recognition, IEEE Transactions on Image Processing 15(7): 1803-1815.

Yu, P., Xu, D., Li, H. \& Zhou, H. (2009). Fingerprint image preprocessing based on whole-hand image captured by digital camera, Computational Intelligence and Software Engineering, 2009. CiSE 2009. International Conference on, pp. 1-4.

Zhang, H., Fritts, J. E. \& Goldman, S. A. (2008). Image segmentation evaluation: A survey of unsupervised methods, Computer Vision and Image Understanding 110(2): 260 - 280.

Zheng, G., Wang, C.-J. \& Boult, T. (2007). Application of projective invariants in hand geometry biometrics, Information Forensics and Security, IEEE Transactions on 2(4): 758-768. 


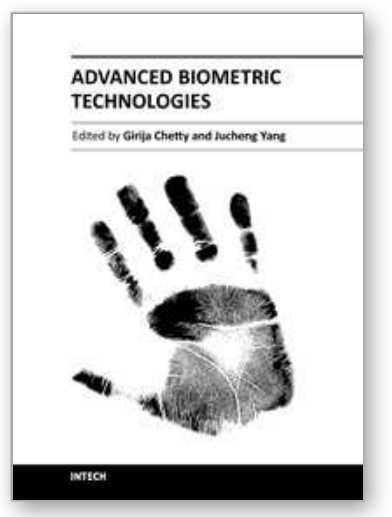

\author{
Advanced Biometric Technologies \\ Edited by Dr. Girija Chetty
}

ISBN 978-953-307-487-0

Hard cover, 382 pages

Publisher InTech

Published online 09, August, 2011

Published in print edition August, 2011

The methods for human identity authentication based on biometrics â€" the physiological and behavioural characteristics of a person have been evolving continuously and seen significant improvement in performance and robustness over the last few years. However, most of the systems reported perform well in controlled operating scenarios, and their performance deteriorates significantly under real world operating conditions, and far from satisfactory in terms of robustness and accuracy, vulnerability to fraud and forgery, and use of acceptable and appropriate authentication protocols. To address some challenges, and the requirements of new and emerging applications, and for seamless diffusion of biometrics in society, there is a need for development of novel paradigms and protocols, and improved algorithms and authentication techniques. This book volume on â€œAdvanced Biometric Technologiesâ€ is dedicated to the work being pursued by researchers around the world in this area, and includes some of the recent findings and their applications to address the challenges and emerging requirements for biometric based identity authentication systems. The book consists of 18 Chapters and is divided into four sections namely novel approaches, advanced algorithms, emerging applications and the multimodal fusion. The book was reviewed by editors Dr. Girija Chetty and Dr. Jucheng Yang We deeply appreciate the efforts of our guest editors: Dr. Norman Poh, Dr. Loris Nanni, Dr. Jianjiang Feng, Dr. Dongsun Park and Dr. Sook Yoon, as well as a number of anonymous reviewers.

\title{
How to reference
}

In order to correctly reference this scholarly work, feel free to copy and paste the following:

Alberto de Santos-Sierra, Carmen Sanchez-Avila, Javier Guerra-Casanova and Aitor Mendaza-Ormaza (2011). Hand Biometrics in Mobile Devices, Advanced Biometric Technologies, Dr. Girija Chetty (Ed.), ISBN: 978-953-307-487-0, InTech, Available from: http://www.intechopen.com/books/advanced-biometrictechnologies/hand-biometrics-in-mobile-devices 1

\section{INTECH}

open science | open minds

\author{
InTech Europe \\ University Campus STeP Ri \\ Slavka Krautzeka 83/A \\ 51000 Rijeka, Croatia \\ Phone: +385 (51) 770447 \\ Fax: +385 (51) 686166
}

\author{
InTech China \\ Unit 405, Office Block, Hotel Equatorial Shanghai \\ No.65, Yan An Road (West), Shanghai, 200040, China \\ 中国上海市延安西路65号上海国际贵都大饭店办公楼 405 单元 \\ Phone: +86-21-62489820 \\ Fax: $+86-21-62489821$
}


www.intechopen.com 
(C) 2011 The Author(s). Licensee IntechOpen. This chapter is distributed under the terms of the Creative Commons Attribution-NonCommercialShareAlike-3.0 License, which permits use, distribution and reproduction for non-commercial purposes, provided the original is properly cited and derivative works building on this content are distributed under the same license. 